livraisons

d'Histoire

de l'Architecture

\section{Livraisons de l'histoire de l'architecture}

13 | 2007

Architectures des établissements d'enseignement supérieur

\title{
L'agrandissement des installations du collège de France sous la monarchie de Juillet
}

Enlarging the premises of the College de France in the reign of Louis Philippe

Die Erweiterungsbauten am Collège de France unter der Monarchie de Juillet

\section{Olivier Lefranc}

\section{(2) OpenEdition}

Journals

Édition électronique

URL : http://journals.openedition.org/lha/410

DOI : $10.4000 /$ /ha. 410

ISSN : 1960-5994

Éditeur

Association Livraisons d'histoire de l'architecture - LHA

Édition imprimée

Date de publication : 10 juin 2007

Pagination : 77-88

ISSN : $1627-4970$

Référence électronique

Olivier Lefranc, «L'agrandissement des installations du collège de France sous la monarchie de Juillet », Livraisons de l'histoire de l'architecture [En ligne], 13 | 2007, mis en ligne le 10 juin 2009, consulté le 24 avril 2019. URL : http://journals.openedition.org//ha/410; DOI : 10.4000//ha.410

Ce document a été généré automatiquement le 24 avril 2019

Tous droits réservés à l'Association LHA 


\section{L'agrandissement des installations du collège de France sous la monarchie de Juillet}

Enlarging the premises of the College de France in the reign of Louis Philippe

Die Erweiterungsbauten am Collège de France unter der Monarchie de Juillet

\section{Olivier Lefranc}

1 Les débuts de la Monarchie de Juillet constituent un moment privilégié pendant lequel sont prises des décisions majeures pour les installations matérielles du Collège de France. En 1831 sont instituées successivement au Collège de France les chaires emblématiques d'«Économie politique » avec Jean-Baptiste Say, de «Législations comparées" avec Eugène Lerminier ${ }^{1}$, d'«Archéologie » - en fait d'«Égyptologie» - créée pour JeanFrançois Champollion, des domaines particulièrement prestigieux dans la France de l'époque où, si l'on reprend des termes ultérieurs d'Ernest Renan, la recherche est « en train de se faire $»^{2}$.

\section{Insuffisance des installations matérielles d'une grande institution}

2 Historiquement, les conditions pratiques de fonctionnement de l'institution fondée par François $I^{\text {er }}$ ont été longtemps sommaires et surtout précaires. Le Collège de France est «basti en hommes », selon l'humaniste du XVI ${ }^{e}$ siècle Étienne Pasquier, propos maintes fois repris ${ }^{3}$ et qui, au fil du temps, a pu être souvent teinté d'amertume. Une recherche approfondie $e^{4}$ montrerait sans doute que les processus de décision de l'Ancien Régime n'étaient pas adaptés à la nécessité de bâtir de la façon suivie qu'aurait demandée cette institution. 
$3 \mathrm{Au}$ «Siècle des Lumières » toutefois, l'élargissement du public fit désirer des amphithéâtres, le goût des sciences expérimentales rendit souhaitables des laboratoires, le besoin d'une efficacité accrue, enfin, accentua la pression pour que soient attribués à certains professeurs des logements sur leur lieu de travail. Aussi Louis XV qui, à la différence de son prédécesseur, avait sensiblement augmenté le nombre des chaires, apparaît-il en 1774 comme le généreux monarque initiateur du projet ${ }^{5}$ de rénovation et d'agrandissement de l'édifice existant. Décidé en 1772 , le projet fut, en dépit de graves vicissitudes ${ }^{6}$ administratives et financières, achevé en $1778^{7}$.

Pendant la Révolution et sous le Premier Empire, le bâtiment se détériore car il est situé dans un environnement urbain trop dense, au bas de la pente nord de la montagne Sainte-Geneviève, humide et escarpée. Aussi, les sommes élevées ${ }^{8}$ consacrées au Collège de France tout au long du Consulat et de l'Empire n'ont-elles eu principalement pour objet que des réparations et des travaux d'entretien ${ }^{9}$. En 1816, en dépit des projets grandioses mais avortés de Napoléon, l'emprise des bâtiments existants n'a en rien évolué par rapport à celle de la fin du XVIII siècle $^{10}$ (ill. 1).

\section{1 : Emprise des bâtiments du Collège de France en 1778, inchangée en 1830}

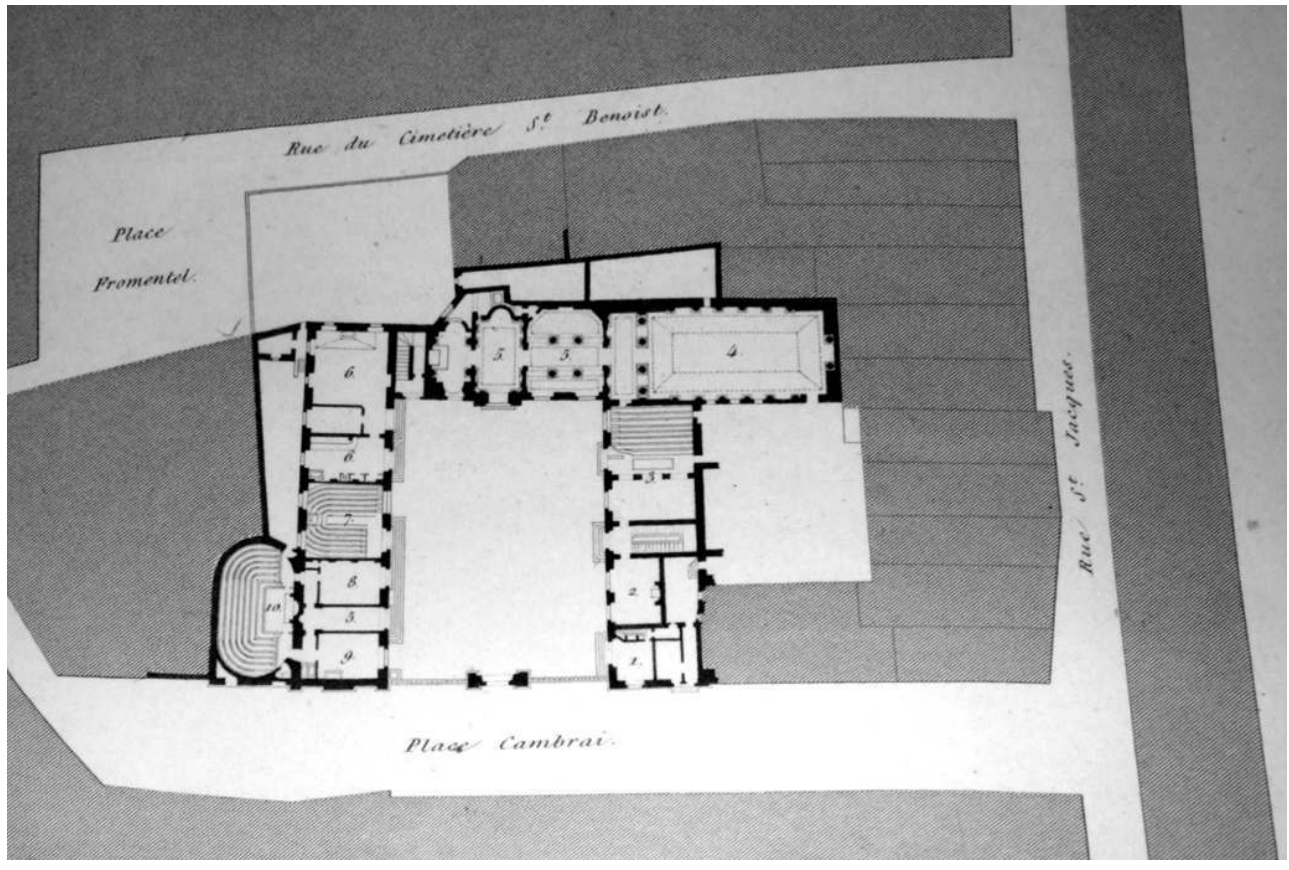

Gourlier, Choix d'édifices publics, Paris, 1845 - 1850, t. III, planche 329

Cl. Olivier Lefranc.

5 Sous la Restauration, il ne manque pas de projets, mais ils n'aboutissent pas de façon sensible malgré les efforts de l'architecte Antoine-Laurent-Thomas Vaudoyer ${ }^{11}$, maintenu dans son poste après l'Empire, puis d'Hippolyte Lebas, son successeur. Â la veille de la chute de Charles X, le gouvernement Polignac envisage même un déménagement de la vieille institution, au motif qu'elle occupe des lieux dont la réfection, inévitable, coûterait un prix jugé exorbitant ${ }^{12}$. 


\section{Efficacité des gouvernants sous Louis-Philippe}

6 La donne change radicalement après les journées de juillet 1830 et l'arrivée de LouisPhilippe au pouvoir. Aujourd'hui, la physionomie de l'édifice historique du Collège de France est certes pour une part de la façade sur la rue des Écoles, celle que Chalgrin a choisie, mais le reste de l'édifice, et plus particulièrement la façade sur la rue SaintJacques, est l'œuvre de la première décennie de la monarchie de Juillet. L'architecte en fut Paul Marie Letarouilly ${ }^{13}$, dynamisé par l'administrateur Silvestre de Sacy ${ }^{14}$, lui-même soutenu puissamment par le ministre puis président du Conseil Adolphe Thiers.

7 L'agrandissement du Collège de France, mené à bien entre 1832 et 1841, consiste en un désenclavement complet, et en un doublement des installations à la faveur de la réorientation opérée vers la rue Saint Jacques. En 1830, en effet, le Collège de France demeure tout aussi enclavé (ill. 1), au bas de la montagne Sainte Geneviève, qu'il l'était sous l'Ancien Régime. Au nord, du côté de l'actuelle rue des Écoles qui ne devait être ouverte qu'en 1854, la place dite de Cambrai est alors sans envergure, limitée par la vaste emprise du Prieuré Saint-Jean de Latran, des Hospitaliers de Jérusalem, longtemps bénéficiaire d'un intangible privilège d'exterritorialité. Á l'ouest, en direction de la rue Saint-Jacques, une dizaine de maisons particulières exclue tout projet important d'extension ; on envisage néanmoins l'acquisition de surfaces de petites dimensions, dans la seule idée d'isoler les installations du Collège d'une proximité trop immédiate des terrains de ces maisons ${ }^{15}$. Â l'est, la configuration des rues et leurs alignements prévus par la Municipalité ${ }^{16}$, s'opposent aux visées que le Collège de France aurait pu nourrir dans cette direction. Au sud, en bordure du Collège du Plessis, c'est-à-dire à la limite de l'actuel Lycée Louis-le-Grand, se trouvent alors deux ruelles tortueuses et peu habitées, la rue du Cimetière Saint-Benoît et la rue Fromentelle. Entre 1800 et 1830, c'est uniquement dans cette direction que des initiatives se manifestent, de façon constante ${ }^{17}$ en faveur d'un agrandissement substantiel. Une telle orientation avait à vaincre non seulement l'obstacle de trois mètres de dénivelé, mais de façon plus déterminante encore celui d'inerties administratives ${ }^{18}$ qui contribuent à expliquer la réorientation ultérieure. Il y avait un projet sérieux vers le sud, celui d'Antoine Vaudoyer, certainement soutenu par l'administrateur du Collège, Lefèvre Gineau. Ce projet ${ }^{19}$, signé par Vaudoyer en 1819, définissait une vaste construction rectangulaire, parallèle au bâtiment du fond de la cour de Chalgrin. Il fut sans nul doute accepté par Silvestre de Sacy successeur de Lefèvre Gineau en 1823, puis repris jusque dans les derniers budgets élaborés pour les années 1831 et 1832 .

\section{L'attribution de moyens financiers}

8 Silvestre de Sacy, sans doute proche des «hommes de juillet» désormais au pouvoir et décidé à ne pas accepter ${ }^{20}$ l'idée d'un déménagement agitée par le dernier gouvernement de Charles X, fut, dès septembre 1830, reçu par Guizot, nouveau ministre de l'Intérieur, dont il obtint ${ }^{21}$ d'emblée, pour l'année à venir, un budget de cinquante mille francs, c'està-dire plus du double des budgets annuels antérieurs ${ }^{22}$.

9 Il n'est pas question encore de construire jusqu'à la rue Saint Jacques. Il s'agit cependant déjà de réaliser une progression dans cette direction. C'est Hippolyte Lebas ${ }^{23}$, successeur 
de Vaudoyer, qui est alors chargé d'élaborer un premier projet d'extension, sur la fraction de l'ancienne implantation du Collège de Cambrai, dite «Petit Cambrai »"

Les choses vont aller vite à partir de ce moment. Le 30 juin 1832, Lebas est remplacé par Paul Marie Letarouilly, avec qui va être préparée l'insertion d'une enveloppe financière, considérable ${ }^{25}$ (650 000 francs) pour le Collège de France, dans la grande loi de 1833 d'Adolphe Thiers prévoyant cent millions de francs pour l'équipement du pays.

11 Le projet, dont Lebas n'a pas commencé la réalisation avant son départ, est confié tel quel à Letarouilly. Á ce premier projet très limité vont succéder non pas un mais deux projets du successeur de Lebas.

12 Jusqu'à juillet 1833 en effet, ni Silvestre de Sacy, l'administrateur, ni son architecte, ne prévoient la possibilité d'acquérir plus qu'une partie des terrains situés derrière les maisons qui bordent la rue Saint Jacques (ill. 1).

Pendant une courte période (septembre 1830 - juillet 1833) ont donc coexisté :

14 - un premier projet Letarouilly, dans lequel l'extension vers la rue Saint Jacques ne dépassait guère l'endroit où se trouve l'actuel portique, sauf à créer une distance suffisante pour éviter les nuisances de voisinage des maisons ;

15 - le vieux projet Vaudoyer auquel on n'avait pas encore renoncé, d'un doublement au sud du bâtiment au fond de la cour de Chalgrin, qui semble n'avoir pas donné lieu à un début de réalisation.

16 Les fouilles récentes, dont rend compte l'ouvrage de Laurent Guyard ${ }^{26}$, prouvent sans doute que les fondations particulièrement robustes trouvées sous le portique s'expliquent par un grand bâtiment rectangulaire que le premier projet Letarouilly aurait voulu situer en cet endroit, et dont la limite se serait trouvée en bordure des terrains des maisons particulières donnant sur la rue Saint Jacques. C'est donc entre septembre 1830 et juillet 1833 que ces fondations auraient été édifiées, mais aucun document d'archives ne vient pour l'instant confirmer cette hypothèse.

\section{Le désenclavement et le doublement des installations}

Le second projet Letarouilly fut conçu en un délai record, à partir de juillet 1833. La loi Thiers du 27 juin 1833 avait été adoptée les 6 et 22 juin par les députés puis les pairs, devant lesquels le ministre avait fait accepter sans difficulté les six cent cinquante mille francs accordés au Collège de France. Si la Madeleine et l'Arc de Triomphe de l'Étoile étaient des édifices «de magnificence " qu'il s'imposait «d'achever», les travaux du Collège de France étaient rangés parmi les "ouvrages d'utilité ", comme les canaux, les routes et les phares, quoique d'une autre manière. Comme en témoignent les débats, ces travaux parurent d'un intérêt indiscutable à la classe politique. Thiers avait de surcroît indiqué qu'ils étaient "de peu de valeur ", c'est-à-dire d'un faible coût, ce qui d'ailleurs lui sera reproché en 1836, car ce n'est qu'en 1836 qu'il expliquera un changement majeur dans les plans, changement en fait intervenu presque immédiatement après le vote de la loi de 1833.

18 L'explication de la réorientation radicale du plan d'agrandissement des installations du Collège de France peut être trouvée dans les effets du vote presque simultané, avec celui du plan d'équipement, d'une nouvelle loi régissant les procédures d'expropriation précédemment définies par un texte de 1810. Cette loi est datée du 7 juillet 1833 et 
quoiqu'elle paraisse aujourd'hui très protectrice des droits des propriétaires, elle fut à l'époque perçue à l'inverse comme tout à fait contraignante ${ }^{27}$.

Le 12 juillet 1833, Silvestre de Sacy écrit à son ministre de tutelle :

J'ai appris seulement depuis deux jours, par l'architecte, que le ministère était dans l'intention d'acquérir toutes les propriétés qui se trouvent sur la place Cambray, entre le Collège et la rue Saint Jacques, et toutes celles qui, de ce côté, séparent le Collège de la même rue, jusqu'au Cimetière Saint Benoit [...]; ce projet aura l'avantage de procurer l'élargissement de la voie publique, d'isoler parfaitement le Collège de trois côtés et de fournir l'emplacement pour deux nouveaux amphithéâtres. Je ne puis donc qu'y donner l'assentiment le plus complet et en désirer l'exécution ${ }^{28}$.

Le rapport du 15 juillet $1833^{29}$ de l'architecte Letarouilly au directeur des travaux publics de Paris, qui fait suite à des instructions du 8 juillet, développe un programme précis conforme à cette orientation, et assorti de considérations esthétiques qu'il respectera ensuite. Il en résultera un plan définitif ${ }^{30}$ (ill. 2) qui, après dix ans de travaux, donnera à la partie ouest du Collège de France son aspect actuel.

III. 2 : Emprise des bâtiments du Collège de France avec les extensions conçues en 1833, réalisées en 1841

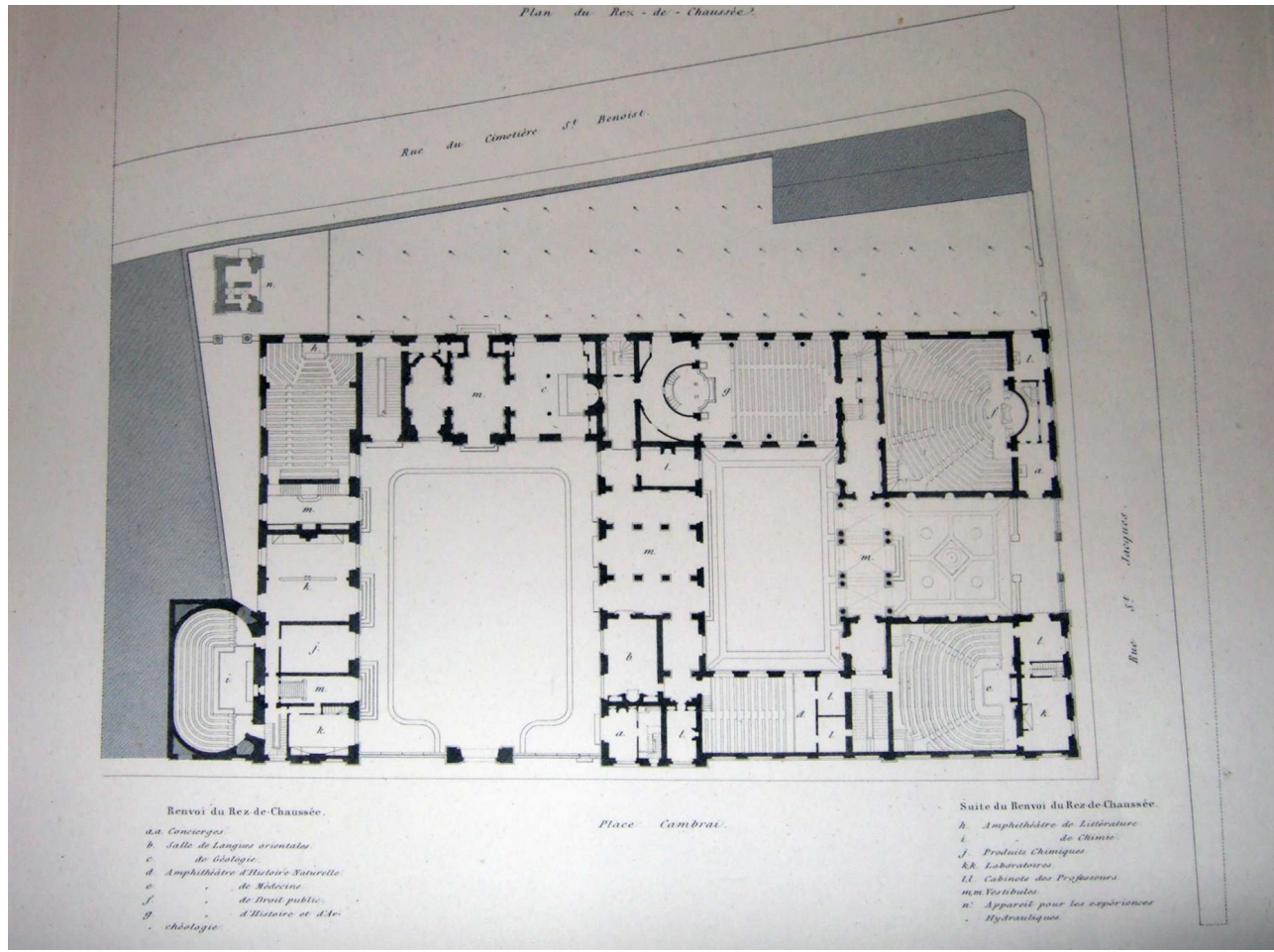

Gourlier, Choix d'édifices publics, Paris, 1845 - 1850, t. III, planche 330

Cl. Olivier Lefranc.

\section{L'implication personnelle d'Adolphe Thiers}

Il importe de souligner ici la part que prit personnellement Adolphe Thiers, ministre des travaux publics puis président du Conseil, aux décisions de désenclavement et de réorientation des installations du Collège de France. Il devait s'en expliquer longuement devant les députés puis les pairs en avril et mai 1836, car il avait été inévitable de retourner devant le Parlement, la modification du premier projet ayant entrâné un 
surcroît de dépenses qui changeait l'ordre de grandeur des coûts. Les prétentions exorbitantes des propriétaires expropriés n'y avaient pas été pour rien, tant il était déjà difficile de garder secrète une opération de ce genre. C'était presque un doublement de l'enveloppe initiale qu'il fallait faire voter.

Thiers donne alors une vision concrète et très personnalisée des choses, qui laisse à penser qu'il aurait fait jouer son poids politique en faveur du changement de nature et de dimensions du projet. Il indique qu'il s'est rendu personnellement sur les chantiers, ce qu'atteste pour le Collège de France un document d'archives du début de $1834^{31}$.

Ceci lui donne l'occasion, dans un échange savoureux avec le très sarcastique député Arago $^{32}$, de décrire l'état défectueux ${ }^{33}$ des anciennes installations. Thiers indique que les parquets sont pourris, les murs salpêtrés, le tout menacé irrémédiablement par un dénivelé de trois mètres, et par les ruissellements qui s'y produisent. C'est ainsi qu'il considère que la réorientation vers la rue Saint-Jacques, dont il s'attribue la paternité, est la seule solution raisonnable, et il réussit à faire voter cette nouvelle loi sans encombre.

Les travaux furent longs, ne serait-ce qu'en raison des difficultés que firent les propriétaires des maisons à acquérir, et ne s'achevèrent qu'en 1841. Le coût total, un peu inférieur à un million deux cent mille francs ${ }^{34}$, est sans doute d'un autre ordre de grandeur que les cent trente mille francs ${ }^{35}$ dépensés pendant les dix premières années de l'Empire, mais reste modéré au regard des huit cent mille francs ${ }^{36}$ indiqués en 1829 pour la seule restauration des installations existantes.

\section{Esthétique et utilité sociale}

Cet effort, sans précédent pour le Collège de France, créa un cadre à la mesure de la grande époque des Jules Michelet (Histoire et Morale, 1838-1852), Adam Mickiewicz ( Langues slaves, 1840-1852), Edgar Quinet (Langues méridionales, 1841-1852). Ce cadre, toutefois, ne rencontra pas l'admiration que l'on aurait pu en attendre, et la presse spécialisée ${ }^{37}$ fut sans pitié pour Letarouilly. Il faut croire qu'en architecture, comme en d'autres domaines, l'austérité rebute l'opinion.

Le Collège de France de Jean-Baptiste Chalgrin, peu cité à la différence d'autres œuvres de ce grand architecte, avait été mal aimé presque dès l'origine, en tout cas dès le début du XIX ${ }^{\mathrm{e}}$ siècle.

27 Chalgrin est mort en 1811 et les modes architecturales ont changé en 1833, lorsque l'architecte Henri de Gisors, dans le rapport ${ }^{38}$ qui dénonce le mauvais état des installations existantes, indique que «le style d'architecture du bâtiment, [construit sous le] règne de Louis XV [...] n'a rien de remarquable».

\section{Caractère durable des options de Chalgrin}

$28 \mathrm{Au} \mathrm{XX}^{\mathrm{e}}$ siècle encore, René Schneider, un auteur dont on connaît le goût pour les grâces du XVIII ${ }^{e}$ siècle finissant, affirme que : «le Collège de France et l'École des Mines sont graves de style comme leur objet et l'époque de d'Alembert $»^{39}$. Et Louis Hautecoeur n'est guère plus bienveillant ${ }^{40}$.

En fait, apparemment insoucieux des modes et particulièrement attachés aux " convenances [propres aux] établissements scientifiques ${ }^{41}$ ", Lebas dans ses dessins ${ }^{42}$ de 1829 repris par Letarouilly, puis Letarouilly dans les projets de 1832 et de 1833, 
conservent scrupuleusement le caractère donné par Chalgrin à l'édifice initial, en portant la façade sud à droite du portail de deux à six, puis sept, puis finalement onze travées, toutes de structure identique à celle des deux premières (ill. 3).

III. 3 : Collège de France : élévation de la façade sur la place Cambrai, aujourd'hui Marcellin Berthelot, prévue et réalisée entre 1833 et 1841

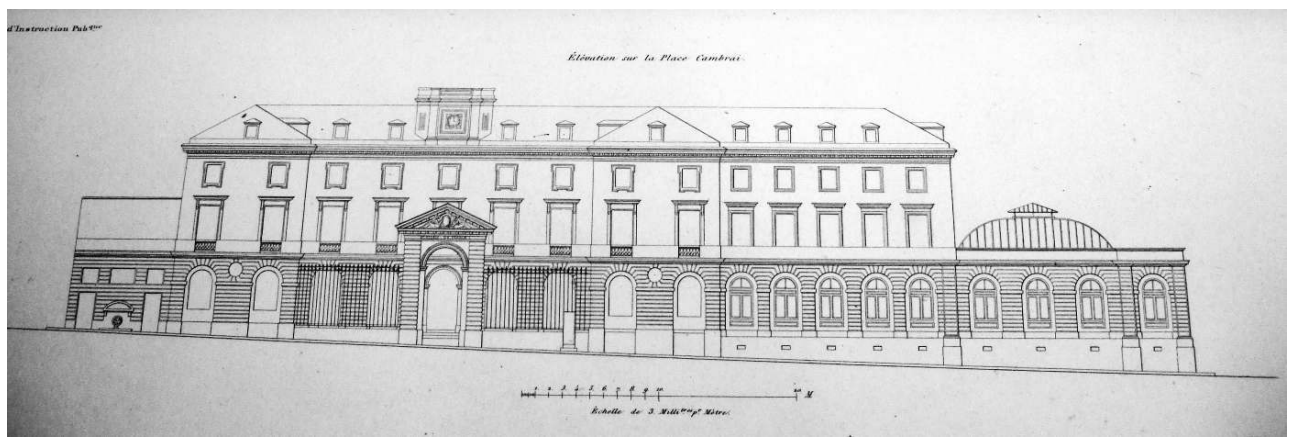

Gourlier, Choix d'édifices publics, Paris, 1845 - 1850, t. III, planche 329

Cl. Olivier Lefranc

Ce sont autant de fenêtres inscrites dans une embrasure en arcade, elle-même ornée de refends, dans la grande tradition classique française. Le parti pris architectural de Letarouilly n'est pas le fait du hasard : il s'agit autant de prolonger l'architecture de Lebas que de rester dans la structure décidée par Chalgrin. L'on ne saurait oublier en effet, ni le goût fondamental de Lebas pour l'arcade, qu'il estimait avoir été inventée par les Étrusques, ni le fait que le même Lebas avait succédé à son oncle Vaudoyer, à la tête de la très importante école dirigée par celui-ci, couramment désignée comme l'«École des Étrusques $»^{43}$.

\section{Filiation rigoureuse des formes}

31 Cette filiation des formes explique aussi le choix par Letarouilly d'un portique à trois arcades, à l'emplacement où il convenait de "distribuer", selon le terme de cet architecte, à l'abri des intempéries, les professeurs et leurs auditeurs entre les quatre amphithéâtres dont allait disposer désormais le Collège. Les trois arcades sont supportées par des colonnes géminées d'un style dépouillé plutôt toscan que dorique, sévère en tout cas. Une continuité forte s'établit ainsi avec les fenêtres des deux amphithéâtres donnant sur la rue Saint-Jacques, quoique ce portique soit en fort retrait au fond de la cour dite aujourd'hui « cour Budé », délimitée par l'avancée de ces deux amphithéâtres (ill. 4 et 5). 
III. 4 : Collège de France : façade sur la rue Saint Jacques : élévation prévue et réalisée par Letarouilly entre 1833 et 1841 , le portique apparaissant au second plan

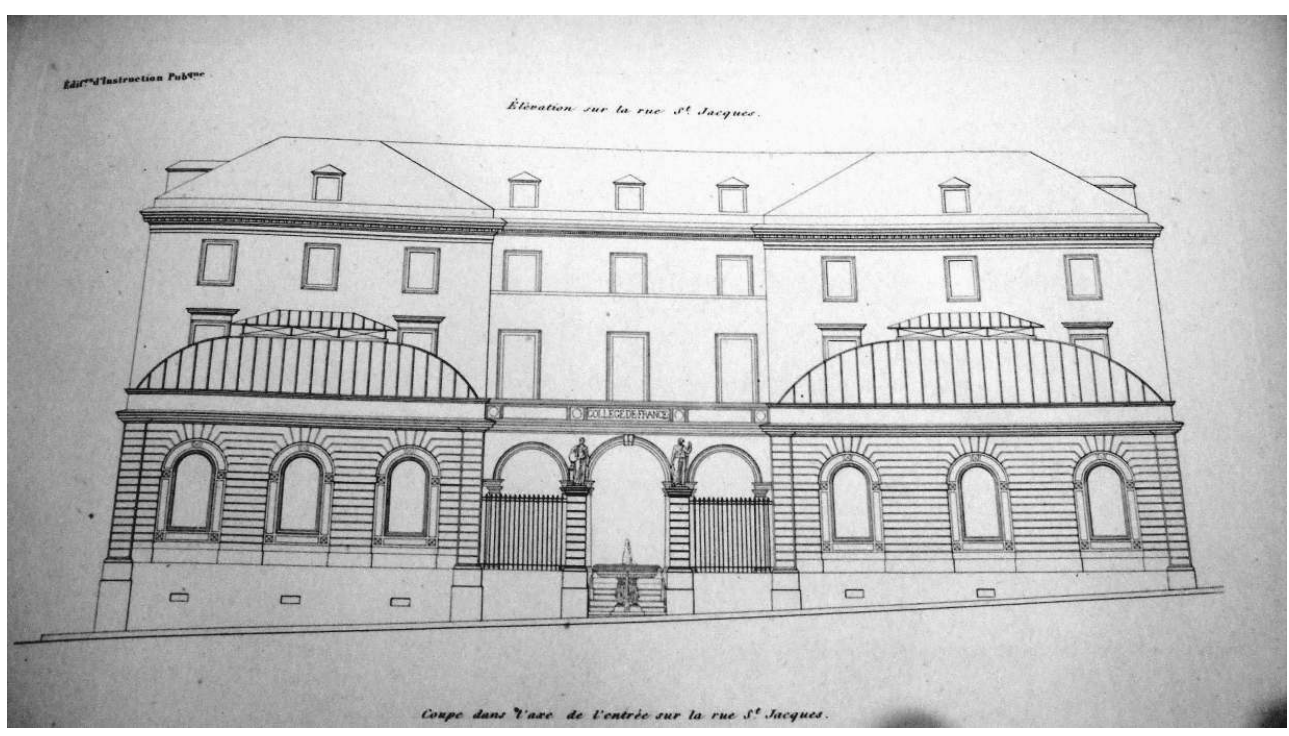

Gourlier, Choix d'édifices publics, Paris, 1845 - 1850, t. III, planche 33

Cl. Olivier Lefranc

III. 5 : Collège de France : vue du portique de Letarouilly de l'intérieur de la cour, en direction de la rue Saint Jacques

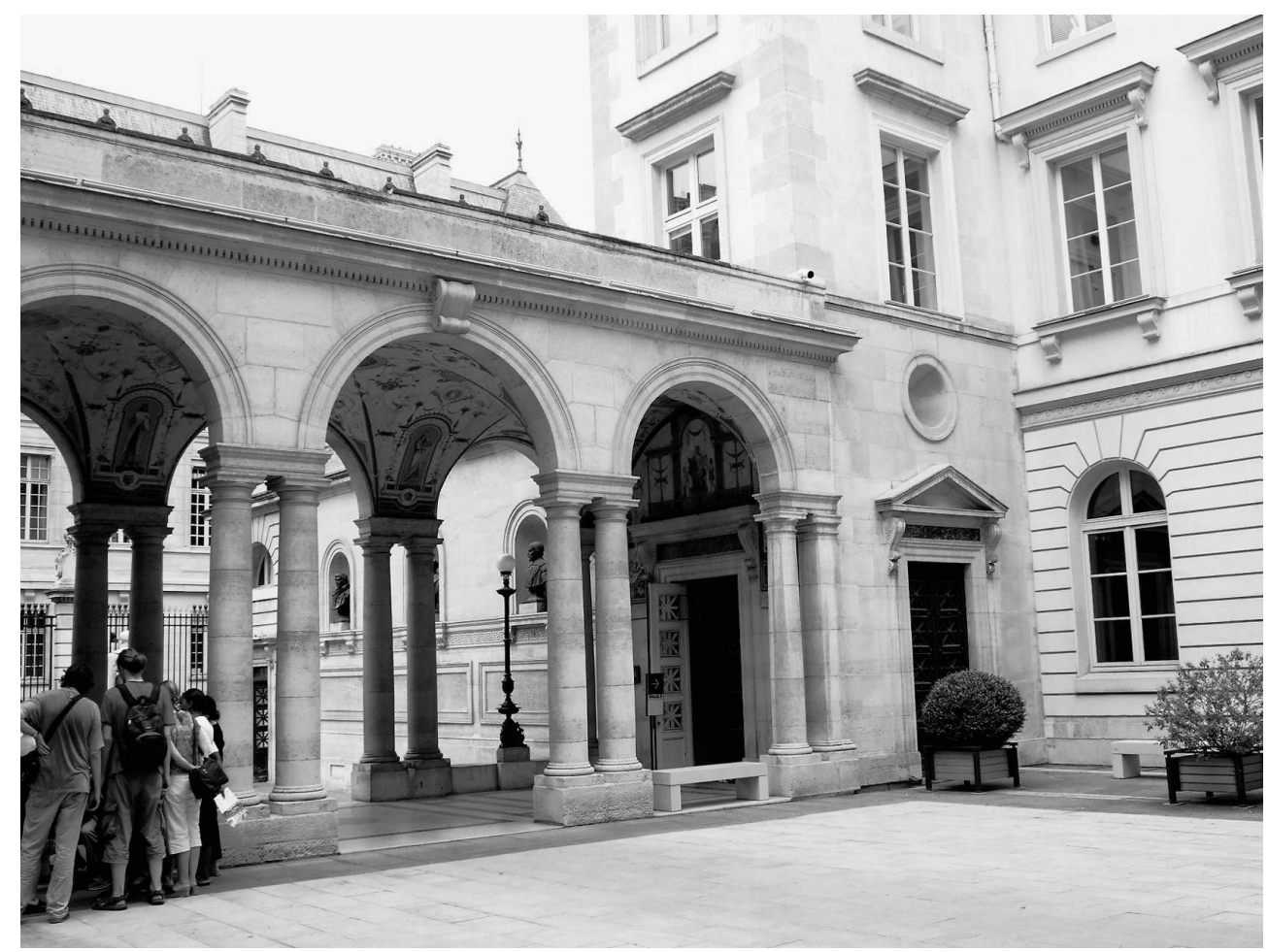

Cl. Olivier Lefranc, juin 2006

32 Quant aux voûtes d'arêtes du portique qui procèdent de la même logique, la même filiation devait conduire à les doter, beaucoup plus tard, d'un décor lui aussi « étrusque ", mais coloré et luxuriant ${ }^{44}$, qu'accompagnent aujourd'hui des luminaires d'un genre floral 
probablement plus tardif. De la même façon, dans les deux cours donnant sur la rue Saint Jacques, les sculptures décoratives ${ }^{45}$ et toute la statuaire sont ultérieures. Voici qui semble prouver (sauf découverte de nouveaux documents d'archives) que tous ces décors n'avaient pas été choisis par Letarouilly. Il s'en était tenu à la sobriété caractéristique de tout le reste de l'édifice et n'avait donné à la Cour Budé, en son centre, qu'une modeste fontaine.

\section{Horizontalité et solidité}

De tout ceci résulte une grande horizontalité, très dépouillée, qui est beaucoup moins évidente dans l'œuvre de Chalgrin. Cette horizontalité s'impose dans l'élévation (ill. 3) de la façade sur la place Cambrai, aujourd'hui place Marcellin Berthelot, même si aujourd'hui de grands arbres en atténuent la perception. C'est pourquoi les représentations que l'on fait traditionnellement de l'ensemble de l'édifice (ill. 6) font apparaître les parties à étages plus hautes qu'elles ne le sont, au-dessus d'une base omniprésente, à arcades et à refends horizontaux.

III. 6 : Le Collège de France après les travaux d'agrandissement, lors des fouilles effectuées sous la chaussée de la rue Saint Jacques

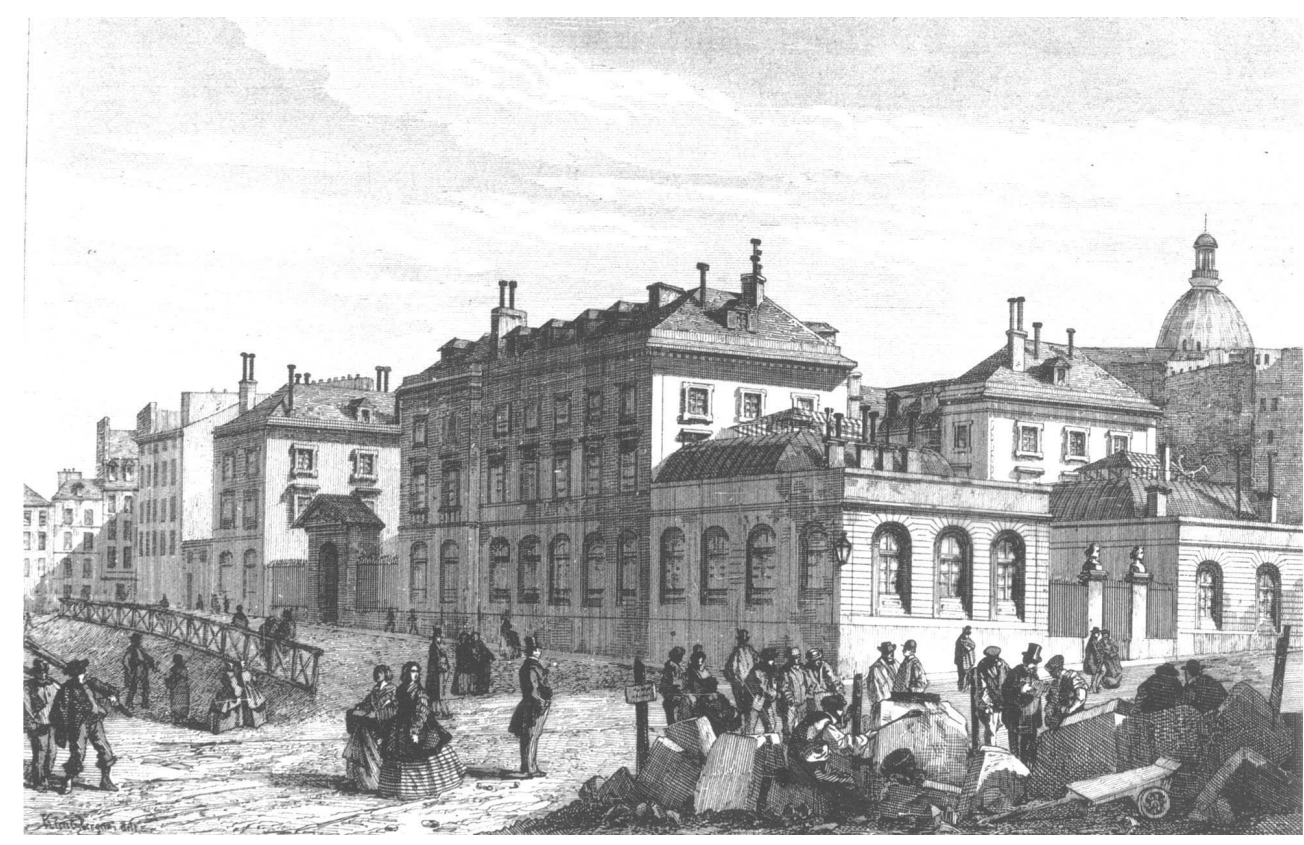

Gravure d'après Kreutzberger, pouvant être datée entre 1842 et 1850, conservée au Collège de France

Cl. Olivier Lefranc

L'effet de solidité du bâtiment, dû à ces assises marquées, constitue la négation sans doute souhaitée alors, de la réputation de fragilité due aux difficultés antérieures.

Il faut souligner, enfin, la grandeur de l'édifice construit par Letarouilly qui ne vise pas alimenter la gloire d'un autocrate, mais à magnifier la seule utilité.

Une grande façade d'un style simple et convenable se développe maintenant aux regards, perpendiculairement à la rue Saint-Jacques et en la remontant, à la place où régnaient autrefois la malpropreté, l'obscurité et le défaut d'air, qui y étaient retenus par d'énormes encaissements de terrains rapportés ${ }^{46}$. 
En juin 1832, Letarouilly avait été nommé « architecte en chef des grands travaux du Collège de France $»^{47}$. Comment ne pas comparer l'ambition des dirigeants d'alors à celle qui, à la fin du $\mathrm{XX}^{\mathrm{e}}$ siècle, a fait admettre la prestigieuse institution au bénéfice des «Grands Travaux de la République »?

\section{NOTES}

1. La grande célébrité que ses idées libérales avancées valurent à Jean-Louis Eugène Lerminier (1803-1857), en charge de cette chaire de droit public comparé, explique que son ralliement en 1838 au gouvernement en place, marqué par sa nomination au Conseil d'État, ait suscité une véritable révolte chez les étudiants; ceux-ci lui imposèrent de se faire remplacer par un suppléant.

2. Ce terme aujourd'hui consacré est par exemple cité par Gilbert Dagron dans Le Collège de France, brochure de présentation, Paris, 1998, p. 19.

3. On peut citer, après bien d'autres, Christophe Charle dans Les Lieux de Mémoire, Pierre Nora (sous la dir. de), Paris, 1986, t. II, 3, p. 404.

4. Des jalons existent pour cette recherche : Alexandre Gady, «Du Collège Royal au Collège de France ", Universités et Grandes Écoles à Paris, Christian Hottin (sous la dir. de), Paris, D.A.A.V.P., 1999, et Marie-Jeanne Tits-Dieuaide, Sur certains aspects du financement de l'Académie des sciences et de quelques autres institutions scientifiques royales en France à l'époque moderne, Bâle, 2005.

5. Plan, Élévation, Coupes et Profils du Collège de France, grand in-folio manuscrit portant la signature de l'architecte Chalgrin, conservé aux Archives du Collège de France.

6. Alexandre Gady, « Du Collège Royal au Collège de France », op. cit.

7. Christian Goudineau et Laurent Guyard, "De Lutèce au Quartier Latin», La Revue de l'Archéologie/Archéologie Nouvelle, n ${ }^{\circ}$ 5, et «Le Collège de France », Laurent Guyard (sous la dir. de), Documents d'archéologie française, $\mathrm{n}^{\circ}$ 95, Paris, 2003.

8. Arch. nat., $\mathrm{F}^{13} 201$, tableau des travaux faits pour la conservation des Bâtiments civils.

9. En témoignent les réunions du conseil des bâtiments civils, dont on peut aujourd'hui commodément analyser les travaux grâce à la base de donnée Conbavil élaborée au centre André Chastel, actuellement en cours d'achèvement et de mise au point.

10. Arch. nat., Cartes et Plans, $\mathrm{F}^{21} 3506$ pièce 1.

11. Antoine Laurent Thomas Vaudoyer (1756-1846), père de l'architecte Léon Vaudoyer (1803-1872).

12. Arch. nat., $\mathrm{F}^{13} 1084$, rapport du 18 août 1829 du directeur des Travaux Publics de Paris au ministre de l'Intérieur. La somme de $800000 \mathrm{~F}$. indiquée par ce rapport est à comparer avec les $1200000 \mathrm{~F}$. qui finalement seront dépensés entre 1830 et 1840 pour une rénovation complète et plus qu'un doublement des surfaces utiles.

13. Paul Marie Letarouilly (1795-1855), architecte du Collège de France après Hippolyte Lebas, de 1832 jusqu'à sa mort en 1855.

14. Antoine Isaac Silvestre de Sacy (1758-1838), orientaliste éminent fut l'un des initiateurs en France de la connaissance de l'arabe. Nommé administrateur du Collège de France en 1823, il le resta jusqu'à sa mort en 1832 . 
15. Après quelques acquisitions, il est encore envisagé, fin 1832, de construire un mur mitoyen entre le Collège de France dans un premier stade de son agrandissement, et les terrains encore privés qui le jouxtent : Arch. nat., $\mathrm{F}^{13} 1084$, rapport du 24 octobre 1832.

16. Arch. nat., Cartes et Plans, N III Seine 1126, pièces 11 et 23.

17. En témoignent notamment les séances des 23 août 1810, 7 septembre 1821, 23 septembre 1828 et 29 mars 1829 du conseil des Bâtiments civils (Conbavil n 15193, 19850, 16849 et 12226$)$.

18. Arch.nat. $\mathrm{F}^{13} 1084$, dossier 2 : le 29 novembre 1828, le préfet de la Seine refuse le projet et crée une commission en charge de l'étudier...

19. Arch. nat., Cartes et Plans, N III Seine 1126, pièce 1.

20. Arch.nat., $\mathrm{F}^{13} 1084$, dossier 4. Lettre de Silvestre de Sacy du 28 décembre 1829.

21. Ibid., lettre de Silvestre de Sacy du 4 septembre 1830.

22. Arch. nat. $\mathrm{F}^{13} 1084$, dossier 4 , lettre du 15 septembre 1830 du directeur des travaux publics au ministre.

23. Hippolyte Lebas (1782-1862), à qui l'on doit, à Paris, Notre-Dame de Lorette et la prison de la Petite-Roquette.

24. Cette fraction de l'ancien Collège de Cambrai (anciennement attribué au Collège de France), non encore reconstruite en 1789 , lui avait été enlevée pendant la Révolution, puis lui avait été restituée sous l'Empire. Les parties les moins délabrées étaient louées à des artisans.

25. Arch. nat., $\mathrm{F}^{13} 201$ et $\mathrm{F}^{21}$ 1370. La somme est d'un nouvel ordre de grandeur par rapport aux 50000 francs déjà consentis et des 10000 ou 20000 francs habituels de l'époque de la Restauration ou même de l'Empire. C'est la promesse de renouveler, sur une durée suffisante, au moins ces 50000 francs annuels. La loi du 27 juin 1833 marque, dans la relation entre le Parlement et le Gouvernement, l'éclosion de ce que l'on appelle aujourd'hui les lois de programme, système financier qui modifie profondément la relation du commanditaire, maître de l'ouvrage, avec l'architecte maître d'œuvre.

26. Laurent Guyard, "Le Collège de France », op. cit., note 7. Cf. également l'opinion du professeur Goudineau, sur le site internet du Collège de France, rubrique « Archéologie ».

27. Alexandre Laya, Études historiques sur la vie privée, politique et littéraire de M. A. Thiers, histoire de quinze ans 1830-1846, Paris, 1846, t. 1, p. 227.

28. Arch. nat., $\mathrm{F}^{13} 1086$, dossier 2 .

29. Arch. nat., $\mathrm{F}^{13} 1084$, dossier 6.

30. Fixé dès l'été 1833, ce nouveau plan ne sera présenté aux députés qu'en 1836, pratiquement identique, ainsi qu'il apparaît dans le document donné aux députés à leur demande, qui est conservé aujourd'hui encore aux Archives de l'assemblée nationale.

31. Arch. nat., $\mathrm{F}^{13} 1084$, dossier 6 , travaux $1833-1835$, rapport de la direction des Bâtiments de Paris.

32. François Arago (1786 -1853), illustre mathématicien et astronome, était alors député des Pyrénées-Orientales et, ardent républicain, siégeait à l'extrême gauche. Partisan d'un déménagement $\mathrm{du}$ Collège de France, il rejoignait paradoxalement les positions du ministère Polignac en 1829.

33. Ce délabrement est dénoncé par un important rapport de l'architecte Gisors du 16 janvier 1833 : Arch. nat., $\mathrm{F}^{21} 1370$.

34. Chiffre officiel publié dans les années qui suivirent (Gourlier, Choix d'édifices publics, Paris 1845-1850, t. III, p. 11). Notons qu'en 1806-1807 l'Arc de Triomphe du Carrousel avait coûté 1000 000 francs, comparaison qu'il n'est pas irrationnel de faire en ce siècle d'un Franc Germinal qui ne connut pratiquement pas de dépréciation.

35. Voir note 8 . 
36. Voir note 11.

37. Revue Générale de l'Architecture et des Travaux Publics, 9 avril 1840, p. 249-250, et octobre 1840, p. 623.

38. Voir note 33.

39. André Michel, Histoire Générale de l'Art, Paris, 1928, t. VII, $2^{\mathrm{e}}$ partie, p. 164.

40. Louis Hautecoeur, Histoire de l'Architecture classique en France, Paris, Picard, 1952, t. IV, p. 219.

41. René Schneider, dans André Michel, ibid.

42. Arch. nat., Cartes et Plans, N III Seine 1126, pièces 17 à 22 et 44.

43. Françoise Largier, Louis-Hippolyte Lebas, architecte historien de l'art, monographie de Muséologie de l'École du Louvre, Paris, 2004, p. 14, 15, 27 et 61, et «Louis Hippolyte Lebas et l'histoire de l'art ", Livraisons d'histoire de l'architecture, $\mathrm{n}^{\circ}$ 9, Paris, 2005, p. 124.

44. En 1891, comme le rappelle Alexandre Gady, « Du Collège Royal au Collège de France », op. cit.

45. Les recherches de Michel-Gasse («Le sculpteur Gustave Germain » dans La lettre du Collège de France, $\mathrm{n}^{\circ} 17$, juin 2006, p. 45) donnent à cet égard des indications déterminantes.

46. Félix Pigeory, Les Monuments de Paris au XIXe siècle, Paris, 1849, p. 251.

47. Arch. nat., $\mathrm{F}^{13} 666$.

\section{RÉSUMÉS}

Le Collège de France, très ancienne institution de recherche et d'enseignement, ne bénéficia pas d'une installation d'une certaine importance avant la fin du XVIII ${ }^{\mathrm{e}}$ siècle. L'édifice conçu par l'architecte Chalgrin, achevé en 1778, était en mauvais état en 1830. Situé au cœur du Quartier Latin à Paris, sur une pente de la montagne Sainte Geneviève, il était enserré dans un tissu urbain trop dense et malsain. Les gouvernements de Louis-Philippe, sous l'impulsion d'Adolphe Thiers, consacrèrent à la rénovation et au développement des constructions existantes, des budgets qui de 1833 à 1841 permirent un doublement des surfaces utiles et un désenclavement sans précédent dans l'histoire de cette institution. Le caractère de l'ensemble ainsi réalisé reste dans la note austère voulue à l'origine par Chalgrin, qui « convenait » à un établissement scientifique d'utilité publique. Le style en est donc sévère, marqué par une grande horizontalité. Ce caractère est le fruit d'une grande continuité avec l'œuvre de Chalgrin, assurée par l'architecte Lebas puis par son successeur Letarouilly qui mena le chantier d'un bout à l'autre.

As a very old institution of higher education and higher research, the College de France did not get significant facilities until the late $18^{\text {th }}$ century. The great architect Chalgrin had been commissioned by Louis XV to design a proper building between 1774 and 1778, but it had not been sturdily built and thus had almost fallen into ruin by 1830 . It was located in the heart of the Paris Quartier Latin, hemmed into a too dense and unhealthy urban area down the Montagne Sainte-Geneviève, where flood caused considerable damage to the floors and walls. Renovating and enlarging the previous buildings was budgeted by the successive governments under the rule of Louis-Philippe, first spurred on by Adolphe Thiers from 1833 to 1841 ; thus the surface area was unprecedentedly doubled and several neighbouring houses were bought to make way for the building. The enlarged building is in a severe style as Chalgrin had intended, that was proper for a state-approved scientific institution, and Classical options are quite respected. Chalgrin first 
made these choices and Lebas, followed by Letarouilly who had it completed, stuck to this initial design.

Erst im späten 18. Jahrhundert wurde das Collège de France, jahrhundertealtes Institut für Forschung und Unterricht, mit umfangreichen Bauten ausgestattet. Das 1778 beendete Gebäude des Architekten Chalgrin war 1830 in schlechtem Zustand. An einen Abhang der Montagne Sainte Geneviève gebaut, war es mitten in dem zu dichten sehr ungesunden Straßengeflecht des Pariser Quartier Latin eingepfercht. Auf Anregung von Adolphe Thiers bewilligten die Regierungen unter König Louis Philippe die Kosten für die Restaurierung und die Änderungen an den vorhandenen Bauten. Die zwischen 1833 und 1841 finanzierten Umbauarbeiten, in der Geschichte des Instituts noch beispiellos, ermöglichten die Verdoppelung der Nutzflächen und die Erschließung des Raumes. Der Gesamtbau berücksichtigt den ursprünglich von Chalgrin gewollten ernsten Charakter, wie es sich für eine gemeinnützige wissenschaftliche Anstalt gehört. Der Stil mit starken geraden Linien entspricht der Kontinuität mit dem Werk Chalgrins, zuerst von dem Architekten Lebas übernommen, dann von dessen Nachfolger Letarouilly, der den Umbau von Anfang bis Ende durchgeführt hat.

\section{AUTEUR}

\section{OLIVIER LEFRANC}

Olivier Lefranc, dont la formation initiale est économique et juridique, est diplômé de l'École du Louvre (2002) avec une spécialité d'iconographie. Diplômé de muséologie en 2003, il consacre sa monographie aux « Projets de réunion du Louvre et des Tuileries entre 1800 et 1850 ». De création récente à l'École du Louvre, le diplôme de recherche appliquée le conduit en 2004 à analyser un manuscrit inédit de 1809, de Percier et Fontaine, donnant à cette date l'état des projets de réunion des deux palais du Louvre et des Tuileries. Á l'heure actuelle, il est doctorant sous la direction de Jean-Michel Leniaud à l'École pratique des hautes études. Le sujet de sa thèse est une recherche sur « Les Palais du Louvre et des Tuileries sous le Consulat et l'Empire ». Adresse électronique : ovlefranc@hotmail.com 